\title{
LES TABLES DE MORTALITE EN L'ABSENCE DE CERTAINES CAUSES DE DECES*
}

\author{
Roland Pressat \\ Institut National D'Etudes Démographiques, Paris, France
}

\begin{abstract}
In this paper the mortality tables in the absence of some causes of death have been the subject of a historical and critical study. Duvillard's famous essay has been exposed and a formula has been established. We have then looked at the works of the followers of Duvillard viz Farr and Westergaard. Finally we have considered the contemporary approaches to the question of mortality caused by accidents, cancer and cardio-vascular diseases.

Résumé-Il s'agit ici d'une étude historique et critique des tables de mortalité en l'absence de certaines causes de décès. On a exposé le célèbre essai de Duvillard et on a établi une formule. Ensuite on a considéré les travaux des continuateurs de Duvillard nommémént Farr et Westergaard. En dernier lieu, on a analysé les recherches contemporaines portant sur la question de la mortalité causée par les accidents, le cancer et les maladies cardio-vasculaires.
\end{abstract}

L'étude des effets de la disparition d'une cause de décès a été inaugurée par l'essai célèbre de Duvillard qui se présente comme la première anticipation scientifique en matière de mortalité.

\section{Les recherches de Duvillard}

Dans son Analyse et tableaux de l'influence de la petite vérole sur la mortalité à chaque âge, et de celle qu'un préservatif tel que la vaccine peut avoir sur la population et la longévité (1806), Duvillard s'est proposé l'étude de maints problèmes en rapport avec l'élimination possible de la "petite vérole," consécutive à la généralisation de la vaccine. Il n'est que de parcourir les nombreux tableaux annexés à son ouvrage, pour se rendre compte de la quantité considérable de situations que notre auteur a entendu décrire; ainsi le tableau 18 donne pour chaque intervalle d'une année d'âge, les probabilités pour les personnes n'ayant pas encore eu la petite vérole.

- "de mourir sans avoir pris la petite vérole"

- "de prendre la petite vérole dans l'année"

- "de mourir de la petite vérole"

- "de prendre la petite vérole et de n'en pas mourir"

- "de mourir de la petite vérole ou de maladie sans avoir pris la petite vérole"

- etc. ...

Les développements mathématiques ayant permis de tels calculs n'occupent pas moins d'une centaine de pages grand format. Nous ne retiendrons que ceux ayant conduit à l'établissement de la table de mortalité en l'absence de variole.

Nous adopterons les notations de Duvillard

$y_{x}$ représente les survivants d'une table de mortalité à l'anniversaire $x$ (ce que nous noterions actuellement $S_{\mathrm{x}}$ ou, dans la notation anglo-saxonne, $l_{\mathrm{x}}$ ). Duvillard distingue dans ces $y_{x}$ personnes.

- les $\gamma_{\mathrm{x}}$ qui mourront tôt ou tard de la variole Ainsi

- les $\epsilon_{\mathrm{x}}$ qui mourront tôt ou tard d'une autre maladie

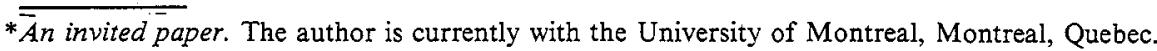




\section{Roland Pressat}

$$
y_{\mathrm{x}}=\nu_{\mathrm{x}}+\epsilon_{\mathrm{x}}(R)
$$

Ce que cherche notre auteur, dans un premier temps, c'est à obtenir la suite des survivants qui définira la table de mortalité en l'absence de variole, et cela à partir des quantités $y_{\mathrm{x}}$ et $\nu_{\mathrm{x}}$.

Duvillard raisonne dans l'intervalle d'âge infiniment petit $(x, x+\dot{d} x)$; en abandonnant l'indice $x$ pour alléger l'écriture, on voit qu'en vertu de la relation (R)

$$
d y=d \nu+d \epsilon
$$

La probabilité de décès "toutes causes" dans l'intervalle $(x, x+d x)$ s'écrit donc,

$$
\underset{y}{d y} \cdot 1
$$

la probabilité en l'absence de la variole se notant:

$$
\frac{d y-d \nu}{y}
$$

conclusions que Duvillard exprime de la façon suivante (p. 22):

"Et de même que la suite $y$, résultant du rapport $\frac{d y}{y}$ est la loi de mortalité générale sous l'influence de la petite vérole, mais dans les temps ordinaires et non dans les temps de calamité, une autre suite $z$, résultant du rapport

$$
\frac{d y-d \nu}{y}-\quad \text { ou } \quad \frac{d z}{z}
$$

sera la loi de mortalité Iorsque l'humanité sera en outre délivrée du fléau de la petite vérole."

Ainsi l'auteur parvient à la relation qu'il cherchait à établir entre $z, y$ et $\nu$ à partir de l'équation différentielle

$$
\frac{d z}{z}=\frac{d y-d \nu}{\underline{y}}
$$

qui lui donne la solution

$$
z=y \exp \left(-\int \frac{\bar{d} \nu}{y}\right)
$$

Cette formule se trouve déjà chez d'Alembert (Duvillard le signale lui-même) ${ }^{2}$. L'apport intéressant de Duvillard concerne l'établissement de formules adaptées aux calculs pratiques.

"Mais de quelque manière que l'on s'y prenne, $v$ et $y$ donnés par l'observation sont tels, que l'expression exacte de $z$ conduira toujours à des calculs très compliqués: or il est important d'avoir une méthode plus simple plus généralement praticable, et qui donne cependant des réșultats très approchés; et l'on va voir que l'on peut atteindre ce but en n'employant que les premières différences finies de $y$ et de $v$ et en prenant le milieu entre les deux extrêmes (p. 23)."

Et ainsi, Duvillard se place dans un intervalle d'âge d'une année $(x, x+1)$ et il notera les variations à l'intérieur de cet intervalle

$$
\Delta y, \Delta y, \Delta z^{3}
$$

Nous voyons alors apparaître une forme de raisonnement maintenant classique,

... Si les $\Delta \nu$ étaient tous morts de la petite vérole à la fin de la xème année on aurait exactement

$$
\frac{\Delta z}{z}=\frac{\Delta y-\Delta \nu}{y}
$$


$\because$ Si au contraire les $\Delta \nu$ sont tous morts au commencement de la xème année, un nombre $\frac{\Delta z}{z} \cdot \Delta \nu$ de ces $\Delta \nu$ seraient morts de maladies dans le courant de l'année, s'ils n'avaient pas été emportés par la petite vérole; et l'on aurait exactement

c'est-à-dire

$$
\frac{\Delta z}{z}=\frac{\Delta y-\left(\Delta \nu-\frac{\Delta z}{z} \cdot \Delta \nu\right)}{y}
$$

$$
\frac{\Delta z}{z}=\frac{\Delta y-\Delta \nu}{y-\Delta \nu}
$$

L'auteur propose de prendre le "milieu" de ces deux résultats, c'est-à-dire leur moyenne arithmétique, ce qui donne

$$
\frac{(\Delta y-\Delta \nu)(2 y-\Delta \nu)}{2 y(y-\Delta \nu)}=\frac{\Delta y-\Delta \nu}{y-1 / 2 \Delta \nu-\frac{1 / 2(\Delta \nu)^{2}}{2 y-\Delta \nu}}
$$

cette dernière expression suggère l'approximation

$$
\frac{\Delta z}{z}=\frac{\Delta y-\Delta \nu}{y-1 / 2 \Delta \nu}(F)
$$

à laquelle on parvient directement en conduisant le raisonnement un peu différemment, sous la forme qui est habituelle maintenant ${ }^{4}$, et qui revient à faire non pas la moyenne arithmétique des deux expressions de $\frac{\Delta z}{z}$ figurant dans la citation de Duvillard, mais la moyenne arithmétique des numérateurs de ces expressions; l'auteur donne aussi, d'ailleurs, cette solution.

Finalement, c'est en exposant le travail de Duvillard que nous avons été amené à établir une formule (F) qui est toujours à la base de l'établissement de tables de mortalité en l'absence d'une cause ou d'un groupe de causes. On l'écrit maintenant, en notant $\mathscr{D}_{1}$ et $\mathscr{D}_{2}$ les décès des deux groupes agissant entre deux anniversaires consécutifs chez $\mathscr{P}$ personnes vivantes au premier des deux anniversaires, et $q_{1}$ le quotient de mortalité relatif au premier groupe (et par conséquent en l'absence du second); on a 5

$$
q_{1}=-\frac{\mathscr{D}_{1}}{\mathscr{S}-\frac{\mathscr{D}_{2}}{2}}
$$

De l'aveu même de Duvillard, cette formule avait déjà été établie; mais peut-être a-t-il eu le mérite de donner le premier exposé systématique de la question et surtout d'offrir avec sa table de mortalité en l'absence de variole le premier exemple d'application.

\section{Effets de l'élimination de la "petite vérole" "}

Virtuose de l'analyse mathématique, Duvillard ne pouvait être que gêné lorsqu'il entendait effectuer des applications, en raison de la rareté ou de l'inadéquation des observations disponibles. Il semble être passé assez allègrement sur les insuffisances de ce qu'il nomme les "faits observés," ce qui rend fort suspecte la valeur de sa célèbre table de mortalité ${ }^{6}$.

C'est à partir de cette table de mortalité qu'il construisit la table de mortalité en l'absence de variole; il lui fallait, pour cela, disposer de données sur la mortalité par variole. Il utilisa principalement des données relatives à Berlin (1757 à 1774), Genève (1580 à 1783), La Haye.

“... réunissant ensuite tous ces résultats et les interpolant, j’ai trouvé la suite ci-après des rapports 
Roland Pressat

$$
\frac{\Delta \nu_{\mathrm{x}}}{\Delta y_{\mathrm{x}}}=r_{\mathrm{x}} "
$$

Ces rapports s'introduisent effectivement dans la formule (F) qui peut s'écrire:

$$
\Delta z=\frac{1-r}{\frac{y}{\Delta y}-1 / 2 r} \cdot z
$$

Nous donnons ci-après quelque extraits des deux tables ainsi que les valeurs correspondantes de $r_{\mathrm{x}}$.

L'élimination de la variole, les autres risques de mortalité ne changeant pas ${ }^{7}$, entraine un gain d'espérance de vie à la naissance de 3.5 ans; en raison de l'importance absolue-de la mortalité par variole entre 1 et 2 ans, le gain est un peu plus élevé à 1 an (3.64 ans), puis il décroit assez rapidement pour être à peu près négligeable dès 20 ans $(0.20 \mathrm{an})$ : c'est que la mortalité par variole décroit très vite avec l'âge, en raison sans doute de l'immunisation d'une fraction croissance des survivants du fait des atteintes antérieures par la maladie.

"mais bien que la dernière suite (survivants en l'absence de mortalité par variole) soit moins rapide que la première, elle n'est cependant pas aussi lente que celle des gens d'élite, des tontiniers, etc. (p. 128)."

Ainsi donc, l'élimination de la variole n'entraine pas, à elle seule, un bouleversement des conditions de mortalité. Dans des populations stationnaires le changement de vie moyenne trouvé par Duvillard équivaudrait au passage du taux brut de mortalité

$$
\mathrm{de}-\frac{1}{28.76}=34.8 \text { pour } 1000 \text { à } \frac{1}{32.26}=31.0 \text { pour } 1000
$$

donc à une baisse de 3.8 p. 1000 soit $11 \%$. Ce résultat se situe dans l'éventail des pourcentages de décès attribués à la variole que l'auteur avait rassemblés (pp. 105 et 106) éventail fort large au demeurant ( 3 à $18 \%$ ). Il est du même ordre de grandeur que les pourcentages communément admis; ainsi E. May écrit ${ }^{8}$

"on a dit aussi que au cours des XVIle et XVIIIe siècles dans l'ensemble de l'Europe, la variole a été responsable de 5 à 10 pour cent de la mortalité générale (p. 94)."

La baisse fut-elle effectivement de cet ordre? A cela, il est impossible de répondre, tout d'abord parce que la pratique de l'inoculation (elle se répandit en France surtout dans le dernier quart du XVIIIème siècle) puis celle de la vaccination (la découverte de Jenner date de 1796), s'installèrent progressivement, en sorte qu'aux effets sur la mortalité provoqués par la généralisation de ces mesures préventives, s'ajoutèrent ceux entrainés par la lente amélioration des conditions générales de vie et d'hygiène. De toute façon, la série des taux de mortalité dont on dispose pour la France, débute avec le commencement du XIXème siècle, donc à une époque où, déjà, la variole n'exerçait plus pleinement ses ravages; quant à la valeur des taux de mortalité, pendant l'Empire, en tant qu'indicateur de l'état normal de la mortalité, on peut en douter. Contentons-nous donc de noter l'évolution pour les premières périodes quinquennales du siècle,

$$
\begin{aligned}
& 1800-1805: 29.3 \text { p. } 1000 \\
& 1806-1810: 26.3 \text { p. } 1000 \\
& 1811-1815: 26.9 \text { p. } 1000 \\
& 1816-1820: 25.3 \text { p. } 1000 \\
& 1821-1825: 24.7 \text { p. } 1000
\end{aligned}
$$

évolution qui ne contredit pas les calculs de Duvillard. 
TABLEAU 1. EXTRAITS DE LA TABLE DE MORTALITE DE DUVILLARD ET DE LA TABLE DE MORTALITE QU'IL EN DEDUISIT PAR SUPPRESSION DE LA MORTALITE PAR VARIOLE

\begin{tabular}{|c|c|c|c|c|c|c|}
\hline \multirow{2}{*}{$\begin{array}{l}\text { Age } \\
x\end{array}$} & \multicolumn{2}{|c|}{ Survivants } & \multicolumn{3}{|c|}{ Espérance de Vie } & \multirow{2}{*}{$\mathrm{r}_{\mathrm{x}}$} \\
\hline & $y_{x}$ & $z_{x}$ & $\left\{y_{x}\right\}$ & $\left\{z_{x}\right\}$ & ifférence & \\
\hline 0 an & 10,000 & 10,000 & 28.76 & 32.26 & 3.50 & 0.08054 \\
\hline $1-$ & 7,675 & 7,842 & 36.35 & 39.99 & 3.64 & 0.24689 \\
\hline 2 ans & 6,718 & 7,094 & 40.43 & 43.16 & 2.73 & 0.31507 \\
\hline $3-$ & 6,247 & 6,749 & 42.44 & 44.34 & 1.90 & 0.32437 \\
\hline $4-$ & 5,987 & 6,559 & 43.26 & 44.61 & 1.35 & 0.30712 \\
\hline $5-$ & 5,832 & 6,440 & 43.40 & 44.42 & 1.02 & 0.27734 \\
\hline $6-$ & 5,730 & 6,359 & 43.16 & 43.98 & 0.82 & 0.24462 \\
\hline $7-$ & 5,658 & 6,299 & 42.70 & 43.40 & 0.70 & 0.21339 \\
\hline $8-$ & 5,602 & 6,250 & 42.12 & 42.74 & 0.62 & 0.18591 \\
\hline $9-$ & 5,555 & 6,207 & 41.48 & 42.03 & 0.55 & 0.16290 \\
\hline $10-$ & 5,511 & 6,166 & 40.80 & 41.31 & 0.51 & 0.14414 \\
\hline--- & $-\cdots--$ & ---- & ----- & ----- & ---- & $---m---$ \\
\hline $20-$ & 5,022 & 5,669 & 34.26 & 34.46 & 0.20 & 0.06058 \\
\hline$--n-$ & ---- & $--\cdots$ & ----- & ---- & --- & --D-- \\
\hline $30-$ & 4,382 & 4,972 & 28.52 & 28.56 & 0.04 & 0.01829 \\
\hline---- & ----- & ----- & --- & ---- & --- & ---N-- \\
\hline $40-$ & 3,694 & 4,198 & 22.89 & 22.90 & 0.01 & 0.00280 \\
\hline --- & $---n$ & $-\cdots-$ & $-\cdots$ & $-\ldots$ & ---- & -- - - \\
\hline $50-$ & 2,971 & 3,377 & 17.23 & 17.23 & 0.00 & 0.00016 \\
\hline---- & $-=--$ & ----- & $-\cdots--$ & ----- & $-m--$ & $----\cdots--$ \\
\hline
\end{tabular}

\section{Les travaux de W. Farr et de H. Westergaard}

Initiateur des calculs de mortalité en l'absence d'une cause de décès, Duvillard a eu des continuateurs jusqu'à l'époque actuelle.

Les travaux de William Farr et de Harald Westergaard, se situant à la fin du XIXème siècle et au début du XXème, renseignent sur la vision que pouvaient avoir, à la veille de progrès spéctaculaires dans la lutte contre la mortalité, des personnalités particulièrement informées des conditions de l'état sanitaire à leur époque.

L'étude de W. Farr que nous analyserons a fait l'objet d'un supplément au 35ème rapport annuel du General Registrar Office; nous nous référons à la traduction française qui en a été donnée dans les Annales de démographie internationale ${ }^{9}$.

Farr pose explicitement le problème des "Effets de l'extinction de quelques maladies particulières sur la durée de la vie" en pleine révolution pastorienne: 
"Il y a un certain nombre de maladies que l'art médical a l'espérance, fondée sur différentes raisons, de pouvoir prévenir. Telles sont la petite vérole, la rougeole, la fièvre scarlatine, la diphtérie, la coqueluche."

Cette fois les diverses hypothèses qu'il convient nécessairement de mettre à la base de tels calculs sont entrevues, mais elles ne sont pas explicitées très clairement ${ }^{10}$.

"Et il est évident que pour juger des effets de la suppression d'une maladie en particulier, il y a trois hypothèses à considérer

$1^{\circ}$ le total des individus vivants ne mourra pas des autres maladies en plus grand nombre qu'auparavant;

$2^{\circ}$ ces autres maladies, par exemple, la fièvre scarlatine et la rougeole acquerront assez de malignité pour compenser, par la perte qu'elles occasionneront, ce qu'on aura gagné d'un autre côté, par exemple, du chef de la petite vérole;

$3^{\circ}$ le gain résultant de la suppression d'une maladie excèdera la perte résultant de l'accroissement des autres ou bien l'inverse aura lieu, en sorte que cette troisième hypothèse se résoudra, soit en un gain partiel, soit en une perte partielle."

Toutefois la suite du texte nous informe plus précisément de la pensée de l'auteur et du choix qu'il a fait.

"Le résultat est que si aucun individu de sexe masculin ne mourait d'une maladie zymotique" quelconque (lère hypothèse), la durée de la vie serait augmentée, quand bien même les individus resteraient sujets aux chances de mortalité provenant des autres maladies."

Le travail du danois $\mathrm{H}$. Westergaārd, Professeur de statistique à l'Université de Copenhague, est bien explicité par son titre: L'horoscope de la population au vingtième siècle.12 C'est donc de vues prospectives générales sur les phénomènes et les structures démographiques dont il s'agit. Notons au passage une attention toute particulière au vieillissement de la population et une pénétrante analyse de ses conséquences.

Westergaard justifie ses anticipations en matière de mortalité par le fait qu'"il n'y a pas de raison de croire que les progrès sanitaires s'arrêteront juste maintenant", argument que l'on retrouve chez tous ceux qui depuis plusieurs décennies, ont établi des prévisions de mortalité en baisse. Cette inertie dans le progrès est fortement justifiée par la phrase suivante: "même si la science n'était plus capable de grandes découvertes, l'effort quiotidien en matière d'hygiène et de médecine suffirait à assurer à l'homme une réduction considérable de la mortalité."

Malgré ces vues très saines sur l'origine des succès dans la lutte contre la mortalité, l'auteur établit ses prévisions dans des hypothèses tout autres, celles de la disparition de telle ou telle cause de décès.

Peu disert sur sa méthode

"Il y a quelques problèmes curieux de statistique théorique posés par ce genre de calculs, mais je n'envisagerai ici que les conséquences pratiques des changements ainsi envisagés,"

Westergaard envisage la disparition possible de divers types de décès et il en mesure le retentissement sur la vie moyenne. ${ }^{13}$

Voici rassemblés les résultats établis par Farr et Westergaard (gains sur l'espérance de vie à la naissance).

Dans tous les cas, ce qui est entrévu est modeste, et Westergaard qui ne doute pourtant pas des changements profonds qui se produiront au cours du XXème siècle (n'envisage-t-il pas une réduction possible du taux de mortalité infantile, l'amenant à $60 \% 0$ à une époque où les valeurs courantes s'établissaient entre 120 et 150?), ne cautionne au mieux, par ses calculs, qu'une vie moyenne future de

49.0 ans chez les hommes

52.8 ans chez les femmes 
TABLEAU 2

\begin{tabular}{|c|c|c|c|}
\hline \multirow[t]{2}{*}{$\begin{array}{l}\text { Causes de décès dont la } \\
\text { disparition est envisagêe }\end{array}$} & \multirow{2}{*}{$\begin{array}{c}\text { W. Farr } \\
\frac{\text { table anglaise } 1861-1870}{S M, e_{0}=39.68}\end{array}$} & \multicolumn{2}{|c|}{$\begin{array}{c}\text { H. Westergaard } \\
\text { table anglaise } 1881-1890 \\
\end{array}$} \\
\hline & & $S M, e_{0}=43.7$ & $\mathrm{SF}, \mathrm{e}_{0}=47.2$ \\
\hline (1) maladies "zymotiques" & 7.09 ans & & \\
\hline (2) phtisie* & 3.28 ans & 3.5 ans & 2.7 ans \\
\hline (3) cancer & 0.20 an & 0.5 an & 1.0 an \\
\hline (4) diarrhée et dysenterie & & 1.1 an & 1.1 an \\
\hline$(2)+(3)+(4)$ & & 5.3 ans & 5.6 ans \\
\hline
\end{tabular}

*chez Westergaard: maladies tuberculeuses

SM - sexe masculin

$\mathrm{SF}$ - sexe féminin

valeurs atteintes en Angleterre-Galles (pays de référence de l'auteur), à la date même . . . . où il rédigeait son étude, et dépassées actuellement d'environ 20 années.

C'est peut-être W. Farr qui dégage le plus nettement la motivation de ce genre de calculs, apparemment peu approprié à la prévision.

"Quiconque a perdu un ami, un frère, un fils par l'effet de quelque maladie, sentira que l'extinction de cette maladie serait pour l'humanité un bienfait dont aucun chiffre ne peut exprimer la valeur. Il y a toutefois quelque chose que l'on peut calculer; c'est l'effet de la suppression d'une maladie quelconque sur la durée de la vie."

\section{Recherches contemporaines}

Ainsi on chercherait, à chaque époque, à isoler les effets des fléaux les mieux reconnus comme les plus redoutés, en déterminant par le calcul les années de vie ainsi perdues qui sont aussi les années de vie que l'on gagnerait si une parade était trouvée.

Duvillard a traité de la sorte le problème de la "petite vérole," Farr et Westergaard ceux du cancer et des maladies infectueuses (avec une attention spéciale à la tuberculose). Les auteurs contemporains isoleront de la même façon la mortalité par accidents (Febvay), par cancer (Henry, Vincent, Wiesler), par maladies cardio-vasculaires (calculs de la Metropolitan Life Insurance Co.).

Le tableau ci-après rassemble quelques uns de ces résultats récents.

L'élimination d'une même cause, ne produit pas partout les même effets; il y a de nombreuses raisons à cela. Tout d'abord, le niveau de la mortalité de référence est variable tant en raison de différences géographiques que de différences temporelles. D'autre part, nous rangeons sous une même appellation des causes qui, selon le choix précis effectué par les auteurs, et selon aussi la qualité des statistiques de causes de décès utilisées, ne forment pas des ensembles rigoureusement comparables.

Malgré cette hétérogénéité, certains traits sont communs aux divers résultats, notamment les gains inégaux de chaque sexe, lors de l'élimination de diverses causes. C'est ainsi que les hommes gagnent beaucoup plus à l'élimination des accidents que les femmes, notamment à l'époque actuelle (gains 3 fois plus élevés): il en va de même pour la tuberculose et plus généralement pour les maladies infectieuses et parasitaires, l'écart étant toutefois moins important; c'est l'inverse qui se produit pour le cancer. 


\section{Roland Pressat}

TABLEAU 3. MODIFICATIONS DE L'ESPERANCE DE VIE A LA NAISSANCE PAR ELIMINATION DE CERTAINES CAUSES DE DECES

\section{Mortalité de réfërence et,} causes de décès supprimés
Wie moyenne selon les tables de référence et gains procurés par la suppression de certaines causes (en années et dixièmes d'année)

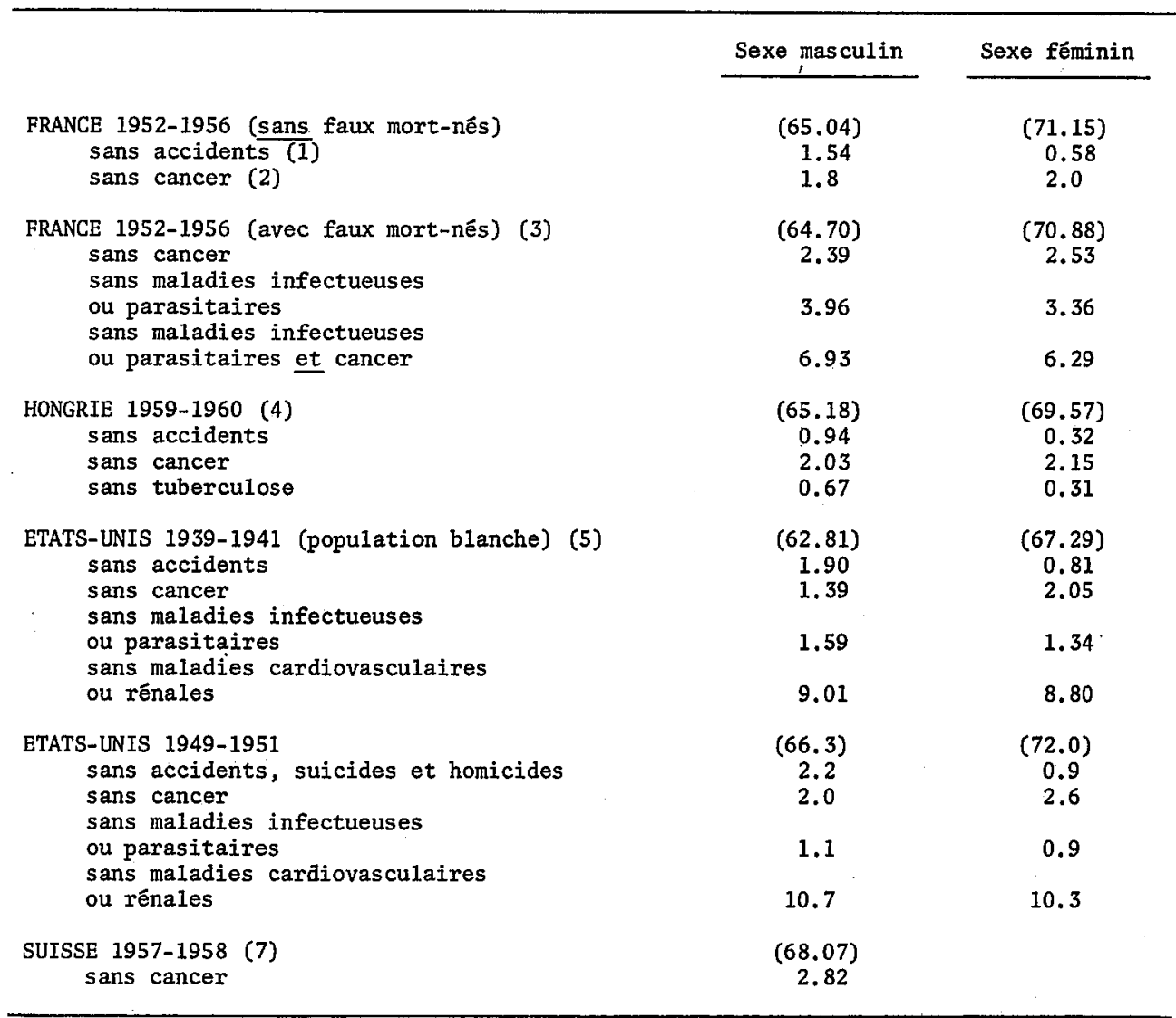

Sources: (1) Etudes statistiques nं̣ 1. janvier-mars 1959.

(2) Louis Henry. Perspectives de population en cas de disparition totale du cancer. Population $n^{\circ} 2.1961$.

(3) Paul Vincent. Sur les effets sur la mortalité, de la disparition éventuelle de certaines causes de décès. Conférence démographique européenne. Strasbourg 30 aoat-septembre 1966.

(4) Agnès Luckacs, Emil Pallós. Quelques aspects du calcul de tables de mortalité par causes de décès. Journal de la Société de statistique de Paris. Avri1, mai, juin 1968.

(5) Dublin, Lotka, Spiegelman. Length of Life. The Ronald Press Company. New York 1949

(6) Barnes Woodhall and Seymour Jablon. Prospects for further increase in average longevity. Geriatrics. Vol. $12 \mathrm{n}^{\circ} 12$. October 1957.

(7) H. Wiesler. L'influence du cancer sur l'ordre de survie. Congrès international de la population. New York. 1961. 
Les progrès les plus notables proviennent naturellement de la suppression des décès par maladies cardio-vasculaires ou rénales, qui forment un pourcentage élevé de ces maladies de dégénérescence qui imposent un terme à la durée de la vie humaine. Mais peut-on alors dire que l'on a supprimé la mortalité causée par ces maladies, si l'on n'a pas remis ce terme en cause? Nous voyons apparaître ici une des limitations commune à toutes les mèthodes de prévision de mortalité; ces prévisions s'établissent toujours dans les limites actuelles de la vie humaine (une centaine d'années), sans que jamais encore on ait imaginé des méthodes qui permettraient une exploration au-delà.

Actuellement d'ailleurs, les statisticiens de la Metropolitan Life Insurance Company ne conduisent plus des calculs avec élimination totale des maladies de dégénérescence; ils ne font plus intervenir que des diminutions de 20.30, ou 50\% (sur les taux probablement).

TABLEAU 4. GAINS D'ESPERANCE DE VIE A 65 ANS PAR DIMINUTION A DIFFERENTS TAUX DE LA MORTALITE PAR MALADIES

CARDIO-VASCULAIRES OU RENALES

\begin{tabular}{|c|c|c|c|c|c|c|c|c|}
\hline \multirow{2}{*}{$\begin{array}{c}\text { Anneé de la mortalité } \\
\text { de référence } \\
\text { (Etats-Unis) }\end{array}$} & \multicolumn{3}{|c|}{ Sexe masculin } & \multicolumn{5}{|c|}{ Sexe féminin } \\
\hline & $20 \%$ & $30 \%$ & $50 \%$ & $20 \%$ & & $30 \%$ & & $50 \%$ \\
\hline 1958 & $1.3 \mathrm{an}$ & & 3.9 ans & 1.4 & an & & & 4.2 ans \\
\hline 1962 & 1.3 an & 2.1 ans & & 1.4 & an & 2.2 & ans & \\
\hline 1964 & 1.24 an & 1.97 an & & 1.30 & an & 2.08 & ans & \\
\hline
\end{tabular}

Sources: Statistical Bulletin. Metropolitan Life Insurance Company. Nov. 1960, Oct. 1964, March 1967.

Mais l'impuissance de la méthode de l'élimination de causes de décès déterminées, pour prévoir l'évolution future de la mortalité, tient aussi à des raisons spécifiques.

Une fois les grandes épidémies conjurées, le recul de la mortalité a moins résulté de la disparition radicale de causes de décès que 'des progrès diffus qui ne cessent de se répandre depuis de nombreuses décennies par l'intermédiare de l'évolution culturelle, économique et sociale. La généralisation d'habitudes hygiéniques, des attitudes nouvelles devant la maladie, de plus grandes possibilités de recourir à des soins sinon toujours pleinement spécifiques, du moins de plus en plus efficaces, constituent sans doute un processus aux effets moins spectaculaires que le resserrement de l'éventail des maux terrestres entrainé par les vaccinations. Mais, la marche de ce processus a quelque chose d'implacable, qui la fait résister, par exemple, à des régressions d'ordre économique (ainsi en période de crise, de chômage).

En d'autres termes, les progrès dans la lutte contre la mortalité passent sans doute, à l'occasion, par la suppression de tel ou tel risque de décès, mais ils résultent aussi de simples consolidations du mécanisme humain, voué de toute façon à la destruction, les survies accordées maintenant à des malades chroniques, dont on n'a pas pu supprimer l'affection dont ils souffrent, peuvant finalement compter davantage en terme global d'années de vie ajoutées, que l'éradication de telle ou telle maladie.

Voilà pourquoi les démarches statistiques précédentes ne sauraient conduire à des prévisions de mortalité. En ce sens, la conclusion de P. Vincent, à son étude Sur les effets 


\section{Roland Pressat}

découle de calculs menés à partir d'observations correspondant à des surmortalités ellesmêmes plus élévées; nous voyons là la preuve de l'impuissance de la méthode à dégager pleinement le "contenu biologique" de la mortalité à partir des classifications nosologiques courantes, cette notion de contenu biologique étant elle-même encore incertaine en l'état de nos connaissances sur les mécanismes d'action de certaines causes de décès.

$\mathrm{Si}$ ce sont les résultats de Bourgeois-Pichat qui ont les apparences les plus satisfaisantes, après 15 ans de progrès sanitaires ils montrent eux aussi certains signes de faiblesse. C'est ainsi que la table de mortalité suédoise 1966-1970 donne pour le sexe féminin, à certains âges, des quotients fort voisins et parfois inférieurs à ceux de la table de mortalité biologique limite, ainsi

à 50 ans 3.36 contre $3.1 \%$

à 60 ans 7.92 contre $8.3 \%$

Mais ce qui est plus troublant, c'est que selon la table suédoise 1966-1970, après la quasi-concordance constatée à 50 et 60 ans, l'écart se creuse, alors que la part des décès endogènes étant de plus en plus forte avec l'avancement en âge, on devrait s'attendre à une sensible identité entre les deux séries; or on enregistre

à 70 ans $24.31 \%$ contre $22.5 \%$

à 80 ans $79.54 \%$ contre $61.1 \%$

En présence de tels résultats, on est amené à se demander si, alors même que l'on saurait bien identifier et isoler la mortalité endogène, il existe bien une seule mortalité endogène, applicable à toutes les populations. On a toute raison d'en douter quand on sait combien varient selon les caractéristiques ethniques, géographiques, climatiques, de comportement (alimentaire par exemple), des populations, la fréquence de certaines atteintes morbides liées au vieillissement (par exemple le cancer, les maladies cardio-vasculaires), et la mortalité qui leur fait suite.

Cette interaction des facteurs biologiques et des facteurs de milieu, jointe à l'ignorance où nous sommes encore du mécanisme de certains processus morbides, font que la notion de mortalité biologique limite ne saurait être que mouvante (dans le temps et dans l'espace), comme les valeurs par lesquelles on entend la caractériser. En définitive, cette notion aide pius à fixer des objectifs de lutte contre la mortalité, compte tenu du savoir et du pouvoir médicaux d'une époque, qu'elle ne permet de bâtir des prévisions. 


\section{Footnotes}

1 Ici et dans la suite, Duvillard a omis le signe moins qui s'introduit du fait que $y_{x}$ est décroissant. Mais, du fait de compensations la formule finale ne s'en trouve pas modifiée. Il est vrai d'ailleurs que Duvillard ne parle pas de probabilités mais de loi de mortalité ainsi qu'on peut l'observer dans la citation qui suit.

2 Auparavant (1760) Daniel Bernoulli avait résolu le même problème, mais en introduisant les fréquences d'apparition de la variole chez les personnes encore indemnes et les taux de mortalité des personnes atteintes, paramètres supplémentaires qui rendaient sa formule difficilement utilisable. Voir à ce sujet M. Noël KARN,

An Inquiry into Various Death-Rates and the Comparative Influence of Certain Diseases on the Duration of Life. Annals of Eugenics, Vol IV (1930-1931).

${ }^{3}$ En conservant toutefois l'indice $\mathrm{x}$ ce que nous ne ferons pas pour alléger l'écriture.

4 Voir par exemple, Roland Pressat, l'Analyse démographique, 2ème edition, page 50.

5 cf. par exemple, Roland Pressat, Principes d'analyse, p. 70.

6 cf. Werner G. Jonckheere. La Table de mortalité de Duvillard. Population No 5 sept-oct. 1965.

7 Cette hypothèse est implicite dans les développements mathématiques ayant conduit à la formule (F); Duvillard ne semble pas l'avoir signalé.

8 I.a médecine. Son passé, son présent, son avenir. Payot. Paris 1957.

9 Première année, 1877, Etude sur la mortalité en Angleterre pendant la période décennale 1861-1870 pp. 85-135 et 207-271.

10 Pour un exposé détaillé de la méthode de Farr, et des légers biais qu'elle entraine, voir Noë Karn. A further Study of Methods of Constructing Life Tables when Certain Causes of Death are Eliminated. Biometrika vol. XXV, 1933.

"Selon Littré: "zymotique: qui offre un caractère de trouble et de dissolution comparée à une fermentation. La variole est une maladie zymotique." Toute imprécise qu'elle soit, cette dénomination recouvre au moins en partie ce que nous considérons comme infectieux. Toutefois, la suite de l'étude, nous montre que M. Farr ne semble pas considérer la phtisie comme une maladie zymotique.

12 Bulletin de l'Institut International de statistique. Tome XVII. Copenhague 1908.

13 Le choix est motivé par le fait qu'il s'agit de causes de décès menaçant l'homme "aux trois stades de la vie".

14 Première conférence européenne de population. Strasbourg 1966.

15 A Bio-Actuarial Approach to Forecasting Rates of Mortality. Proceedings Centenary Assembly of the Institute of Actuaries. 1950.

16 cf. Demographic and Actuarial Aspects of Ageing, with Special Reference to England and Wales. Journal of the Institute of Actuaries. Vol. 90, part III. N $\mathrm{N}^{\circ} 386,1964$.

17 La mortalité, phénomène biométrique. Population, $\mathrm{N}^{\circ} 1$, janvier-mars 1952

18. La mortalité des vieillards. Population, $\mathrm{N}^{\circ} 2$, avril-juin 1951.

is Etude biométrique de la mortalité par vieillissement. Relations de type allométrique entre les causes de décès. Congrès mondial de la population, Volume 1, Rome 1954.

20 Toutefois le résultat de Tabah et Sutter, pour l'ensemble des deux sexes en Norvège, surpasse légèrement le résultat correspondant de Bourgeois-Pichat pour la France (77.7 ans contre 77.2 ans).

Received August, 1974. 